\title{
The Spanish Journal of Psychology
}

Date of delivery: $28-06-2018$

\begin{tabular}{l|l|l} 
Journal and vol/article ref: & sjp & S113874161800029X
\end{tabular}

Number of pages (not including this page): 7

This proof is sent to you on behalf of Cambridge University Press. Please print out the file and check the proofs carefully. Please ensure you answer all queries.

Please EMAIL your corrections within

2 days of receipt to:

\section{Ana Montero \\ <psyjourmanaging@psi.ucm.es>}

Please download the relevant copyright transfer form below and return to

journalscopyright@cambridge.org

Standard Copyright Form: https://www.cambridge.org/core/services/aop-file-manager/file/575eb6a2b8cf5823190221b0

Open Access Copyright Form: https://www.cambridge.org/core/services/aop-file-manager/file/575eb6c2b109e94515d4e0ee

Authors are strongly advised to read these proofs thoroughly because any errors missed may appear in the final published paper. This will be your ONLY chance to correct your proof. Once published, either online or in print, no further changes can be made.

NOTE: If you have no corrections to make, please also email to authorise publication.

- The proof is sent to you for correction of typographical errors only. Revision of the substance of the text is not permitted, unless discussed with the editor of the journal. Only one set of corrections are permitted.

- Please answer carefully any author queries.

- Corrections which do NOT follow journal style will not be accepted.

- A new copy of a figure must be provided if correction of anything other than a typographical error introduced by the typesetter is required.

- If you have problems with the file please email psyjourmanaging@psi.ucm.es

Please note that this pdf is for proof checking purposes only. It should not be distributed to third parties and may not represent the final published version.

Important: you must return any forms included with your proof. We cannot publish your article if you have not returned your signed copyright form

\section{Please do not reply to this email}

NOTE - for further information about Journals Production please consult our FAQs at http://journals.cambridge.org/production_faqs 


\section{Author Queries}

QA $\quad$ The distinction between surnames can be ambiguous, therefore to ensure accurate tagging for indexing purposes online (eg for PubMed entries), please check that the highlighted surnames have been correctly identified, that all names are in the correct order and spelt correctly. 


\title{
Impact of Prenatal Depressive Symptoms on Postpartum Depressive Symptoms: Mediation Effect of Perinatal Health
}

\author{
QA Fábio Silva ${ }^{1}$, Ana Conde $^{1}$ and Raquel Costa ${ }^{2}$ \\ 1 Universidade Portucalense (Portugal) \\ 2 Universidade Europeia / Laureate International Universities (Portugal)
}

\begin{abstract}
To analyze the mediation effect of perinatal health on the association between prenatal depressive symptoms and postpartum depressive symptoms 180 women filled the Edinburgh Postnatal Depressive Scale (EPDS) at 35 weeks of gestation and two months after childbirth. Perinatal health data was collected during the first 4 days after childbirth, using the Optimality Index. $25.6 \%$ of the mothers-to-be presented clinically significant depressive symptoms, and of these, $80.4 \%$ still show clinically significant depressive symptoms at 2-months postpartum. Prenatal depressive symptoms predict higher postpartum depressive symptoms. Additionally, results also showed that the effect of prenatal depressive symptoms on postnatal depressive symptoms is not mediated by perinatal health. Mothers-to-be with prenatal depressive symptoms seem to be at risk for postnatal depression, even when perinatal health is not compromised. This highlights the importance of early screening of prenatal depressive symptoms in order to promote an early intervention on women's mental health, leading to a better transition to parenthood and to a decrease of the burden of this public health problem on children and families.
\end{abstract}

Received 15 February 2017; Revised 15 June 2018; Accepted 19 June 2018

Keywords: perinatal health, prenatal depressive symptoms, postpartum depressive symptoms, screening.

Depression is one of the most common psychological problems reported during pregnancy and the postpartum period (Lee \& Chung, 2007). The reported prevalence varies from $15 \%$ to $29.2 \%$, in the prenatal period (e.g., Flynn, 2005; Goodman \& Tyer-Viola, 2010; Sidebottom, Hellerstedt, Harrison, \& Hennrikus, 2014; Teixeira, Figueiredo, Conde, Pacheco, \& Costa, 2009) and from $4.6 \%$ to $16.5 \%$ in the postnatal period (e.g., Andersson, Sundström-Poromaa, Wulff, Åström, \& Bixo, 2006; Felice, Saliba, Grech, \& Cox, 2004; Segre, O'Hara, Arndt, \& Stuart, 2007). Despite several moments of assessment were used in the different studies, there is a general consent that the prevalence of depressive symptoms is higher in the prenatal period compared to the postnatal period (Andersson, et al., 2006; Canário \& Figueiredo, 2017; Faisal-Cury \& Menezes, 2012; Figueiredo \& Conde, 2011; Goodman \& TyerViola, 2010; Sidebottom, et al., 2014; Underwood, Waldie, D'Souza, Peterson, \& Morton, 2016).

A relevant question to consider when depressive symptoms are addressed during the perinatal period is whether or not there is continuity in symptomatology

\footnotetext{
Correspondence concerning this article should be addressed to Raquel Costa. Universidade Europeia. Escola de Saúde. Estrada da Correia, 53, Carnide Universidade Europeia, Laureate International Universities, Lisboa 1500-210. Portugal.

E-mail: rqlcosta@gmail.com

Funding information: Fundação Bial, Ref. 157/12.
}

from the prenatal to the postnatal period. Some studies show that women with clinically significant depressive symptoms after childbirth were already depressed during pregnancy (e.g., Heron et al., 2004; Robertson, Grace, Wallington, \& Stewart, 2004; Sidebottom et al., 2014; Underwood et al. 2016). In fact, consistent results suggest that depressive symptoms during pregnancy are the most consistent predictor of postpartum depressive symptoms (Heron, et al., 2004; Kim, Hur, Kim, Oh, \& Shin, 2008; Robertson, et al., 2004) compared to other factors such as obstetric factors (Johnstone, Boyce, Hickey, Morris-Yates, \& Harris 2001; Nielsen, Videbech, Hedegaard, Dalby, \& Secher, 2000). Nonetheless, perinatal outcomes, such as high number of visits to the antenatal care clinic, premature contraction, hyperemesis, episiotomy, caesarean wounds, breast pain (Josefsson, et al., 2002), gestational hypertension and / or eclampsia and breastfeeding difficulties (Koutra et al., 2016), also seem to be related to depressive symptoms in the postpartum period. On the other hand, evidence additionally support the association between prenatal depressive symptoms and negative perinatal outcomes, namely

How to cite this article:

Silva, F., Conde, A., \& Costa, R. (2018). Impact of prenatal depressive symptoms on postpartum depressive symptoms: Mediation effect of perinatal health. The Spanish Journal of Psychology, 21. eXX. Doi:10.1017/sjp.2018.29. 
prematurity (Liou, Wang, \& Cheng, 2016) and low birth weight (Alder, Fink, Bitzer, Hösli, \& Holzgreve, 2007).

Given this evidence on the association of prenatal depressive symptoms and perinatal health, as well as on the association of perinatal health and postnatal depressive symptoms, an important issue to explore is the potential mediation effect of the perinatal health on the association between perinatal depressive symptoms and postnatal depressive symptoms.

Considering the negative impact of both low-levels and clinically significant postpartum depression symptoms for child development (Conners-Burrow et al., 2016), it is important to clarify its determinants. In this study, we intend to analyze the association between prenatal depressive symptoms and postpartum depressive symptoms, exploring the mediation effect of perinatal health.

\section{Method}

\section{Sample}

The sample included 180 pregnant women. Most participants were aged between 30 and 39 years old, were married or in a non-marital partnership, had monthly incomes higher than $1000 €$ and more than 12 years of education (see Table 1). Considering obstetric outcomes, $25.3 \%$ of women referred the occurrence of previous abortion, most of them were primiparous (62.8\%), had a natural conception $(96.7 \%$ ) and a vaginal delivery with

Table 1. Mother's Socio Demographics $(N=180)$

$(\%)$

\begin{tabular}{llc}
\hline Age (years) & $<20$ & 0.6 \\
& $20-29$ & 32.2 \\
& $30-39$ & 62.8 \\
& $\geq 40$ & 4.4 \\
Marital status & Married/Co-habitation & 73.7 \\
& Single/Divorced & 26.3 \\
Monthly Income $(€)$ & $<1000$ & 29.9 \\
& $>1000$ & 70.1 \\
Education (years) & $<12$ & 48.9 \\
& $>12$ & 51.1 \\
Optimality Index & SMB & $M(S D)$ \\
& PPMSDTM & $5.65(2.84)$ \\
& P & $3.44(1.11)$ \\
& ND & $12.44(2.78)$ \\
& CMPD & $6.36(0.95)$ \\
& OPP & $2.82(0.49)$ \\
& & $30.32(5.87)$
\end{tabular}

Note: SMB = Social and medical background; PPMSDTM = Present pregnancy, maternal status, diagnostic and therapeutic measures; $\mathrm{P}=$ Parturition; $\mathrm{NC}=$ Neonatal condition; $\mathrm{CMPD}=$ Condition of the mother prior to discharge from birth facility or from provider care; OPPP = Total score of Optimality Index. epidural (42\%). The high majority of pregnant women did not signalize substances consumption $(7.8 \%$ of the participants smoked, but no cases of alcohol or drug consumption were referred).

\section{Measures}

\section{Socio-demographic questionnaire}

Participants completed a self-report questionnaire composed by 43 items, developed for this study in order to collect information about age, race, nationality, marital status, professional status, education, number of children and medical and obstetric history. Information related to the consumption of substances, such as drugs and tobacco, were also collected.

\section{Edinburgh Postnatal Depression Scale (EPDS) (Cox, Holden, E Sagovsky, 1987)}

EPDS is a self-report questionnaire that consists of 10 items scored in a 0 to 3 Lickert Scale, designed to measure postpartum depression (intensity of depressive symptoms in the previous 7 days). In order to determine the prevalence of depression during the perinatal period, optimal cut-off points were established for pregnancy $(\geq 9)$ and the postpartum period $(\geq 7)$ (Tendais, Costa, Conde, \& Figueiredo, 2014). In the present study EPDS showed good internal consistency $(\alpha=.782$ at pregnancy and $\alpha=.779$ after childbirth). It has been used in several studies both with pregnant and postpartum women, namely in Portugal (Areias, Kumar, Barros, \& Figueiredo, 1996; Figueiredo \& Conde, 2011; Figueiredo, Pacheco, \& Costa, 2007; Teixeira et al., 2009; Gorman et al., 2004). The EPDS Portuguese version has shown good internal consistency $(\alpha=.85)$ (Figueiredo et al., 2007).

\section{Optimality Index (Murphy \& Fullerton, 2006)}

The Optimality Index is a clinimetric instrument that provides information about five different perinatal domains computed through the sum of all the occurrences in each item: (1) The social and medical background (SMB: Marital status; ethnic minority; smoking; alcohol; drug use; pre-pregnancy body mass index; age; preexisting major chronic disease; inter-pregnancy interval between index pregnancy and previous viable birth; previous preterm delivery; previous intrauterine fetal death; previous caesarean section; previous $<51 \frac{1}{2}$ pounds at birth; other serious antepartum complications). (2) The present pregnancy, maternal status, diagnostic and therapeutic measures used (PPMSDTM: Intrauterine fetal demise; domestic violence; other serious antepartum conditions / complications; prenatal care: initiation in first trimester and minimum 5 visits; amniocentesis; nonstress test / contraction stress; medication use). (3) Parturition 
(P: Period of time between first digital examination following rupture of membranes and birth; amniotic fluid; induction/augmentation of labor; amniotomy; oral or injectable medication during first or second stage of labor; epidural analgesia for labor and/or birth; fetoscope; fetal heart rate abnormalities; presence of a support person during labor; non-directed pushing; delivery occurred in the place originally intended; nonsupine position at birth; presentation at birth; instrumental (vaginal) delivery; cesarean section; episiotomy; $1^{\text {st }}$ or $2^{\text {nd }}$ degree laceration of perineum or perineal tissue; $3^{\text {rd }}$ or $4^{\text {th }}$ degree extension of either and episiotomy or a $1^{\text {st }}$ or $2^{\text {nd }}$ degree laceration; medication during third stage of labor; skin-to-skin contact; placental retention; postpartum hemorrhage; blood transfusion; other intrapartum complications). (4) Neonatal condition (NC: Estimate of gestational age; birth weight; Apgar score at 5 minutes; transfer to high risk neonatal care setting; congenital anomalies; birth trauma or other serious medical problem; breastfeeding; perinatal death). And (5) Condition of the mother prior to discharge from birth facility or from providers care (CMPD: Fever while mother remains in the birth setting or provider diagnosis of infectious process or major complications; prescription medication for conditions newly identified in intrapartum or postpartum period; maternal mortality). The instrument provides an optimality measure in the perinatal care, so the best condition with the minimum interventions is intended, regarding the mother and the child. The concept of optimality is defined as the absence of perinatal problems and the need of apply corrective interventions. When an intervention is used, the score is considered non optimal, even if it is necessary to intervene in a specific complication. The Portuguese version resulted from a two-step translation process (translation and retroversion to Portuguese language) of the original instrument, made by the Portuguese research team.

\section{Procedures}

This longitudinal study is part of a wider project, "Contributions of Parent-Infant Psychophysiology during Dyadic Interactions to Child Development", financed by BIAL foundation (157/12) involving 387 couples. Despite the initial sample of the project involved 387 couples, the fathers were not included in the present study and only the 180 mothers who completed all the assessments were included in the analyses. This study was evaluated and approved by the National Committee for Data Protection and by the ethics committee of the hospital.

The recruitment of the pregnant women was implemented in the main city hospital and in 13 primary care units. All mothers-to-be were contacted during a routine medical appointment at 35 weeks of gestation. Exclusion criteria were not being able to read and write in
Portuguese and having fetus with congenital problems. The objectives of the study were clearly explained to eligible participants, and information regarding the study methods and procedures was provided. After the informed consent was signed, the first data collection was initiated. In the first assessment moment (35 weeks of gestation), sociodemographic and clinical data, as well as information about the use of substances (alcohol, drugs and tobacco) was collected. The Portuguese version of EPDS was also filled in at this time point in order to assess prenatal depressive symptoms. The perinatal health data collected from medical reports during the first four days after childbirth, using the Optimality Index. Repeated measures of mothers' depressive symptoms were obtained 2-months after childbirth using the Portuguese version of EPDS.

\section{Data analysis}

Data were analyzed using the Statistical Package for the Social Sciences (SPSS), version 20.0. Descriptive statistics were used for the sociodemographic characterization of the sample and for determining the proportion of women with clinically significant depressive symptoms during pregnancy and postpartum. Paired sample $t$-test was conducted to analyses differences on depressive symptoms between pregnancy and postpartum. Several separate linear regression analyses were performed to analyze the association between prenatal depressive symptoms on postpartum depressive symptoms, exploring the potential mediation effect of perinatal health. Following the premises of the Baron and Kenny (1986) mediation model, four conditions were tested: (1) Variation on prenatal depressive symptoms account for variations in postnatal depressive symptoms (Path g, Figure 1); (2) variation on prenatal depressive symptoms account for variations in perinatal health (Path a1, b1, c1, d1, e1, f1, Figure 1); (3) variation in the perinatal health account for variations in postnatal depressive symptoms (Path a2, b2, c2, d2, e2, f2, Figure 1); and (4) a previously significant relation between prenatal depressive symptoms and postnatal depressive symptoms is significantly reduced or no longer significant when the perinatal health is added to the model (Path $\mathrm{g}^{\prime}$, Figure 1). If path $\mathrm{g}$ is reduced to zero, then prenatal depressive symptoms can be considered a single mediator, whereas if path $\mathrm{g}$ is not reduced to zero multiple mediating factors may exist (Baron \& Kenny, 1986).

\section{Results}

\section{Depressive symptoms at pregnancy and postpartum}

Self-reported measures implemented during pregnancy showed similar levels of depressive symptoms at pregnancy $(M=6.07 ; S D=4.17$; $\operatorname{Min}=0 ;$ Max. $=19)$ 


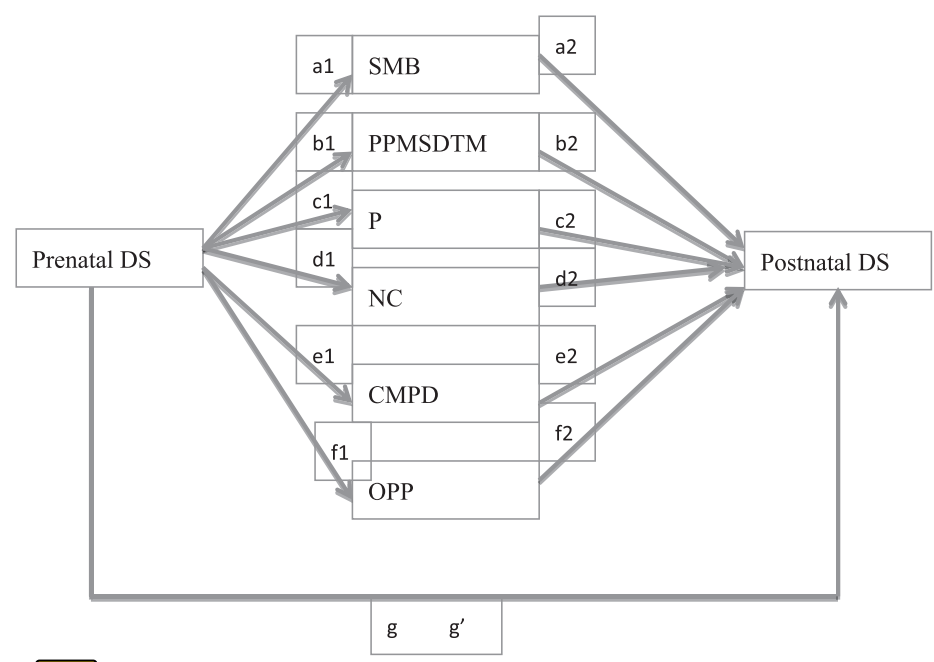

Figure 1. Mediation Model. $\Omega$

$\mathrm{SMB}=$ Social and medical background; PPMSDTM = Present pregnancy, maternal status, diagnostic and therapeutic measures; $\mathrm{P}=$ Parturition; $\mathrm{NC}=$ Neonatal condition; $\mathrm{CMPD}=$ Condition of the mother prior to discharge from birth facility or from provider care; OPPP = Total score of Optimality Index.

and postpartum $(M=5.93 ; S D=3.5 ; \operatorname{Min}=0 ;$ Max. $=19)$ $(t=.582, p=.562)$. Nevertheless, $25.6 \%$ of the mothersto-be presented clinically significant depressive symptoms (EPDS > =9), and of these, $80.4 \%$ still show clinically significant depressive symptoms at 2-months postpartum (EPDS > = 7). An incidence of 33 new cases (18.3\%) were observed after childbirth, with a total prevalence of $38.9 \%$ (EPDS > = 7).

\section{Test of Mediation Model}

Condition 1: Prenatal depressive symptoms as predictor of postpartum depressive symptoms

The linear regression model showed that prenatal depressive symptoms predicted higher postpartum depressive symptoms $\left(R^{2}=.441 ; F=140.609 ; p<.001\right)$.

Condition 2: Prenatal depressive symptoms as predictor of perinatal health

The linear regression model showed that prenatal depressive symptoms predicted two dimensions of the perinatal health: (1) PPMSDTM $\left(R^{2}=.016 ; F=4.257\right.$; $p=.040)$ and (2) OPP $\left(R^{2}=.016 ; F=4.025 ; p=.048\right)$. (See table 2)

\section{Condition 3: Perinatal health as predictor of postpartum depressive symptoms}

Several linear regression models were performed in order to analyze the potential predictive effect of the different domains of perinatal health, - SMB, PPMSDTM, P, ND, CMPD, OPP - on postpartum depressive symptoms. The results showed that none of the perinatal health domains predicted postpartum depressive symptoms (see Table 3). These analyses excluded perinatal health outcomes as potential mediators of the effect of prenatal depressive symptoms on postpartum depressive symptoms.

\section{Condition 4: Test of mediation effect}

Attending to the rejection of the conditions 2 and 3, the condition 4 was not tested because the mediation effect of perinatal health was excluded by the previous conditions.

\section{Discussion}

Considering the negative impact of both low-levels and clinically significant postpartum depression symptoms on child development (Conners-Burrow et al., 2016) and, consequently, on the family functioning, it is important to identify the pathways through which different risk factors can lead to depression after childbirth. This study analyzed the association between prenatal depressive symptoms and postpartum depressive symptoms, exploring the mediation effect of perinatal health. The results show that prenatal depressive symptoms predict higher postpartum depressive symptoms. Additionally, show that, although prenatal depressive symptoms predict worse perinatal health outcomes, the association between prenatal depressive symptoms and postnatal depressive symptoms is not mediated by perinatal health.

A relevant question to consider when depressive symptoms are addressed during the perinatal period is whether or not there is continuity from the prenatal and postnatal periods. In this study, the vast majority of women with clinically significant symptoms during 
Table 2. Prentive Depressive Symptoms as Predictor of Perinat Health

\begin{tabular}{lllllrr}
\hline Model & $R^{2}$ & $F$ & Sig $F$ & $\beta$ & $t$ & $p$ \\
\hline SMB & .008 & 2.112 & .147 & .089 & 1.453 & .147 \\
PPMSDTM & .016 & 4.257 & .040 & .126 & .063 & .040 \\
P & .000 & .057 & .812 & .015 & .062 & .812 \\
NC & .004 & 1.009 & .316 & .054 & .316 \\
CMPD & .003 & .561 & .455 & .126 & .749 & .455 \\
OPPP & .016 & 4.025 & .046 & .006 & .048 \\
\hline
\end{tabular}

Note: $\mathrm{SMB}=$ Social and medical background; PPMSDTM = Present pregnancy, maternal status, diagnostic and therapeutic measures; $\mathrm{P}=$ Parturition; $\mathrm{NC}=$ Neonatal condition; $\mathrm{CMPD}=$ Condition of the mother prior to discharge from birth facility or from provider care; OPPP $=$ Total score of Optimality Index.

Table 3. Perinatal Health as Predictor of Postpartum Depressive Symptoms

\begin{tabular}{lllllrr}
\hline Model & $R^{2}$ & $F$ & Sig $F$ & $\beta$ & $t$ & $p$ \\
\hline SMB & .000 & .005 & .945 & -.005 & -.069 & .945 \\
PPMSDTM & .000 & .050 & .823 & -.017 & -.224 & .823 \\
P & .007 & 1.278 & .260 & -.084 & -1.130 & .260 \\
NC & .007 & 1.201 & .275 & .082 & .096 & .275 \\
CMPD & .002 & .374 & .542 & .049 & .611 & .542 \\
OPPP & .001 & .133 & .716 & -.027 & -.364 & .716 \\
\hline
\end{tabular}

Note: SMB = Social and medical background; PPMSDTM = Present pregnancy, maternal status, diagnostic and therapeutic measures; $\mathrm{P}=$ Parturition; $\mathrm{NC}=$ Neonatal condition; $\mathrm{CMPD}=$ Condition of the mother prior to discharge from birth facility or from provider care; OPPP $=$ Total score of Optimality Index.

pregnancy had still clinically significant symptoms after childbirth which is in accordance with the idea that most cases of postpartum depression are not in fact new cases, but are actually cases of depression during pregnancy that remained untreated after childbirth. This data reinforced data from other studies (Evans, Heron, Francomb, Oke, \& Golding, 2001), showing the importance of early screening and treating depression symptoms even during pregnancy and not only after childbirth, preventing the vulnerability trajectories on women's mental health during the perinatal period.

Our results also show that the prevalence of prenatal clinically significant depressive symptoms is lower compared to the prevalence of postpartum clinically significant depressive symptoms. This is apparently inconsistent with previous evidence, namely our own previous findings, showing that the prevalence of clinically significant depression symptoms during pregnancy is usually higher than at postpartum (Andersson, et al., 2006; Felice, et al., 2004; Figueiredo \& Conde, 2011; Goodman \& Tyer-Viola, 2010; Sidebottom, et al., 2014; Teixeira et al., 2009). Nonetheless, this apparent inconsistency may be due to the use of the EPDS cutoffs recently considered to be optimal for establishing clinically significant symptoms during pregnancy (9) and the postpartum period (7), when compared to a gold-standard interview in a Portuguese sample (Tendais at al., 2014). These new EPDS cut-offs are lower in the postpartum compared to those used in other studies, which may explain this disparity. This was the first study in Portugal that has analyzed the EPDS criterion validity against a gold standard in women during pregnancy and postpartum, and our study is, as far as we know, the first using these optimal cut-offs. The studies involving mental health self-report measures should consider evidence based appropriate clinical cut-offs not only for screening but also for research purposes.

The results of this study strength the idea that one of the best predictors of postpartum depressive symptoms is the presence of depressive symptoms during pregnancy (Heron, et al., 2004; Kim et al., 2008; Robertson, et al., 2004), namely when compared to other factors such as obstetric factors (Johnstone et al., 2001; Nielsen et al., 2000). Thus, screening depression during pregnancy should be an established aim of health facility services for an early intervention on women's mental health preventing the negative impact of the chronicity of depression during the transition to parenthood on child development and on family environment.

On the other hand, an effect of prenatal depressive symptoms on overall perinatal health outcomes, 
particularly on perinatal outcomes concerning to the current pregnancy, was also found. This result supports and extends evidence on the association between prenatal depressive symptoms and negative perinatal outcomes, namely prematurity and low birth weight (Alder et al. 2007). Nevertheless, none of the perinatal outcomes (namely social and medical background; present pregnancy, maternal status, diagnostic and therapeutic measures; parturition; neonatal condition; condition of the mother prior to discharge from birth facility or from provider care; global perinatal health) assessed in the present study, were significant predictors of postpartum depressive symptoms. This apparently contradicts results of previous studies showing that perinatal outcomes, such as high number of visits to the antenatal care clinic, premature contraction, hyperemesis, episiotomy, caesarean wounds, breast pain (Josefsson, et al., 2002) as well as gestational hypertension and/or eclampsia and breastfeeding difficulties (Koutra et al., 2016) are associated with depressive symptoms in the postpartum period. However, these perinatal health outcomes in previous studies were analyzed individually, whereas in this study, these outcomes were combined in specific domains, and analyzed globally as an index of perinatal health outcomes.

The inexistence of a predictor effect of perinatal outcomes on postpartum depressive symptoms implies that the effect of prenatal depressive symptoms on postnatal depressive symptoms is not mediated by perinatal health. This is particularly important to acknowledge, since it suggests that mothers-to-be with depressive symptoms are still at risk for postpartum depresion symptoms, even when perinatal health is not compromised. This was an unexpected result, attending to the fact that previous studies have reported an association between prenatal depressive symptoms and perinatal health (Alder et al., 2007) and between perinatal health and depressive symptoms in the postpartum period (Josefsson, et al., 2002). Nonetheless, those studies were conducted analyzing prenatal depressive symptoms or postnatal depressive symptoms, but not both. This study is, as far as we know, the first one reporting data from both prenatal depressive symptoms and postnatal depressive symptoms and analyzing several perinatal health outcomes, collected using reliable and extensive medical records, as mediators.

There are some limitations to point out, namely the fact that the final sample was composed of 180 mothers (out of the 387 initial mothers) that have completed all the assessment moments, and these might eventually be those with lower depression symptoms, which may constitute a bias. On the other hand, self-reported measures for depression symptoms were used to collect information, instead of clinical interviews. Nonetheless, when considering clinically significant symptoms for the EPDS, we have used the optimal cut-offs established for the Portuguese sample using a clinical interview. Despite lhis limitations, this study points out the importance of early screening for depressive symptoms during the prenatal period in order to promote women's perinatal mental health and diminish the chronicity of depressive symptoms during the transition to parenthood and, consequently the burden for children and families. New health antenatal care policies should include women's mental health screening as a fundamental target in order to promote, prevent and protect maternal and perinatal health.

\section{References}

Alder J., Fink N., Bitzer J., Hösli I., \& Holzgreve W. (2007). Depression and anxiety during pregnancy: A risk factor for obstetric, fetal and neonatal outcome? A critical review of the literature. Journal of Maternal and Fetal Neonatal Medicine, 20(3), 189-209. https: / / doi. org /10.1080/14767050701209560

Andersson L., Sundström-Poromaa I., Wulff M., Åström M., \& Bixo M. (2006). Depression and anxiety during pregnancy and six months postpartum: A follow-up study. Acta Obstetricia et Gynecologica Scandinavica, 85(8), 937-944. https:/ / doi.org/10.1080/00016340600697652

Areias M. E. G., Kumar R., Barros H., \& Figueiredo E. (1996). Comparative incidence of depression in women and men, during pregnancy and after childbirth. Validation of the Edinburgh Postnatal Depression Scale in Portuguese mothers. The British Journal of Psychiatry, 169, 30-35. https:/ / doi.org/10.1192/bjp.169.1.30

Baron R. M., \& Kenny D. A. (1986). The moderator-mediator variable distinction in social psychological research: Conceptual, strategic, and statistical considerations. Journal of Personality and Social Psychology, 51, 1173-1182. https:/ / doi.org/10.1037/0022-3514.51.6.1173

Canário C., \& Figueiredo B. (2017). Anxiety and depressive symptoms in women and men from early pregnancy to 30 months postpartum, 35(5), 431-449. Journal of Reproductive and Infant Psychology. https: / / doi. org/10.1080/02646838.2017.1368464

Conners-Burrow N. A., McKelvey L., Perry D., Whiteside-Mansell L., Kraleti S., Mesman G., ... Kyzer A. (2016). Low-level symptoms of depression in mothers of young children are associated with behavior problems in middle childhood. Maternal and Child Health Journal, 20(3), 516-524. https:/ / doi. org/10.1007/s10995-015-1849-0

Cox J. L., Holden J. M., \& Sagovsky R. (1987). Detection of postnatal depression. Development of the 10-item Edinburgh Postnatal Depression Scale. The British Journal of Psychiatry, 150(6), 782-786. https:/ / doi. org/10.1192/bjp.150.6.782

Evans J., Heron J., Francomb H., Oke S., \& Golding J. (2001). Cohort study of depressed mood during pregnancy and after childbirth. British Medical Journal, 323(7307), 257-260. https://doi.org/10.1136/ bmj.323.7307.257 
Faisal-Cury A., \& Menezes P. R. (2012). Antenatal depression strongly predicts postnatal depression in primary health care. Revista Brasileira de Psiquiatria, 34, 446-450. https: / / doi.org/10.1016/j.rbp.2012.01.003

Felice E., Saliba J., Grech V., \& Cox J. (2004). Prevalence rates and psychosocial characteristics associated with depression in pregnancy and postpartum in Maltese women. Journal of Affective Disorders, 82(2), 297-301. https://doi.org/10.1016/j.jad.2003.11.011

Figueiredo B., \& Conde A. (2011). Anxiety and depression in women and men from early pregnancy to 3-months postpartum. Archives of Women's Mental Health, 14(3), 247-255. https: / / doi.org/10.1007/s00737-011-0217-3

Figueiredo B., Pacheco A., \& Costa R. (2007). Depression during pregnancy and the postpartum period in adolescent and adult Portuguese mothers. Archives of Womens' Mental Health, 10, 103-109. https:/ / doi.org/10.1007/s00737-0070178-8

Flynn H. A. (2005). Epidemiology and phenomenology of postpartum mood disorders. Psychiatric Annals, 35(7),

544-551. https://doi.org/10.3928/0048-5713-20050701-12

Goodman J. H., \& Tyer-Viola L. (2010). Detection, treatment, and referral of perinatal depression and anxiety by obstetrical providers. Journal of Women's Health, 19(3), 477-490. https: / / doi.org/10.1089/ jwh.2008.1352

Gorrman L. L., O'Hara M. W., Figueiredo B., Hayes S., Jacquemain F., Kammerer M. H., ... Sutter-Dallay A. L. (2004). Adaptation of the structured clinical interview for DSM-IV disorders for assessing depression in women during pregnancy and post-partum across countries and cultures. The British Journal of Psychiatry, 184(S46), s17-s23. https:/ / doi.org/10.1192/bjp.184.46.s17

Heron J., O'Connor T. G., Evans J., Golding J., Glover V., \& ALSPAC Study Team (2004). The course of anxiety and depression through pregnancy and the postpartum in a community sample. Journal of Affective Disorders, 80(1), 65-73. https: / /doi.org/10.1016/j.jad.2003.08.004

Johnstone S. J., Boyce P. M., Hickey A. R., Morris-Yates A. D., \& Harris M. G. (2001). Obstetric risk factors for postnatal depression in urban and rural community samples. Australian and New Zealand Journal of Psychiatry, 35(1), 69-74. https://doi.org/10.1046/j.1440-1614.2001.00862.x

Josefsson A., Angelsiöö L., Berg G., Ekström C.-M., Gunnervik C., Nordin C., \& Sydsjö G. (2002). Obstetric, somatic, and demographic risk factors for postpartum depressive symptoms. Obstetrics \& Gynecology, 99(2), 223-228. https://doi.org/10.1016/S0029-7844(01)01722-7

Kim Y. K., Hur J. W., Kim K. H., Oh K. S., \& Shin Y. C. (2008). Prediction of postpartum depression by sociodemographic, obstetric and psychological factors: A prospective study. Psychiatry and Clinical Neurosciences, 62(3), 331-340. https:/ / doi.org/10.1111/j.1440-1819.2008.01801.x
Koutra K., Vassilaki M., Georgiou V., Koutis A., Bitsios P., Kogevinas M., \& Chatzi L. (2016). Pregnancy, perinatal and postpartum complications as determinants of postpartum depression: The Rhea mother-child cohort in Crete, Greece. Epidemiology and Psychiatry Sciences, 27(3), 244-255. https:/ / doi.org/10.1017/S2045796016001062

Lee D. T., \& Chung T. K. (2007). Postnatal depression: An update. Best Practice \& Research Clinical Obstetrics \& Gynaecology, 21(2), 183-191. https://doi.org/10.1016/j. bpobgyn.2006.10.003

Liou S.-R., Wang P., \& Cheng C.-Y. (2016). Effects of prenatal maternal mental distress on birth outcomes. Women and Birth, 29(4), 376-380. https: / / doi.org/10.1016/j. wombi.2016.03.004

Murphy P. A., \& Fullerton J. T. (2006). Development of the Optimality Index as a new approach to evaluating outcomes of maternity care. Journal of Obstetric, Gynecologic, E Neonatal Nursing, 35(6), 770-778. https:/ / doi.org/10.1111/j.1552-6909.2006.00105.x

Nielsen D., Videbech P., Hedegaard M., Dalby J., \& Secher N. (2000). Postpartum depression: identification of women at risk. BJOG: An International Journal of Obstetrics \& Gynaecology, 107(10), 1210-1217.

Robertson E., Grace S., Wallington T., \& Stewart D. E. (2004). Antenatal risk factors for postpartum depression: a synthesis of recent literature. General Hospital Psychiatry, 26(4), 289-295. https:/ / doi.org/10.1016/j. genhosppsych.2004.02.006

Segre L. S., O'Hara M. W., Arndt S., \& Stuart S. (2007). The prevalence of postpartum depression: the relative significance of three social status indices. Social Psychiatry and Psychiatric Epidemiology, 42(4), 316-321. https: / / doi. org/10.1007/s00127-007-0168-1

Sidebottom A. C., Hellerstedt W. L., Harrison P. A., \& Hennrikus D. (2014). An examination of prenatal and postpartum depressive symptoms among women served by urban community health centers. Archives of Women's Mental Health, 17(1), 27-40. https:/ /doi.org/10.1007/ s00737-013-0378-3

Teixeira C., Figueiredo B., Conde A., Pacheco A., \& Costa R. (2009). Anxiety and depression during pregnancy in women and men. Journal of Affective Disorders, 119(1), 142-148. https:/ / doi.org/10.1016/j.jad.2009.03.005

Tendais I., Costa R., Conde A., \& Figueiredo B. (2014). Screening for depression and anxiety disorders from pregnancy to postpartum with the EPDS and STAI. The Spanish Journal of Psychology, 17, E7. https://doi. org/10.1017/sjp.2014.7

Underwood L., Waldie K., D'Souza S., Peterson E. R., \& Morton S. (2016). A review of longitudinal studies on antenatal and postnatal depression. Archives of Women's Mental Health, 19(5), 711-720. https://doi.org/10.1007/ s00737-016-0629-1 